\title{
A MEDITAÇÃO NO ESPORTE DE ALTO RENDIMENTO: REVISÃO SISTEMATIZADA DA LITERATURA ${ }^{1}$
}

\author{
Vinícius Demarchi Silva Terra \\ Universidade Federal de São Paulo, São Paulo, São Paulo, Brasil \\ Felipe Falcoski \\ Universidade Federal de São Paulo, São Paulo, São Paulo, Brasil \\ Ricardo da Costa Padovani \\ Universidade Federal de São Paulo, São Paulo, São Paulo, Brasil \\ Emilson Colantonio \\ Universidade Federal de São Paulo, São Paulo, São Paulo, Brasil
}

\begin{abstract}
Resumo
Este artigo analisa as evidências de estudos observacionais que avaliaram o desempenho esportivo de atletas sob programas de meditação. A pesquisa foi realizada nas bases: LILACS, SCIELO, PubMed $e$ MEDLINE. Qualificaram-se as evidências pela escala PEDro e tabularam-se os protocolos utilizados para o treino, os parâmetros avaliados e os efeitos da meditação na performance e as modalidades esportivas. Treze trabalhos foram analisados e apresentaram associação positiva entre meditação e aumento da performance esportiva, entre os quais houve predominância de abordagens comportamentais e avaliação dos preditores cognitivos do desempenho esportivo individual. Apesar dos resultados positivos, os protocolos de meditação utilizados demonstraram inconsistências com os fundamentos do treinamento esportivo.

Palavras-chave: Meditação. Desempenho atlético. Esporte.
\end{abstract}

\section{Introdução}

A meditação consiste em uma prática milenar de origem oriental, com inserções filosóficas na ioga e no budismo (LEVINE, 2000), e seus múltiplos significados variam conforme a técnica abordada. Sua ocidentalização ocorreu no final da década de 1950, com um importante polo difusor em São Francisco (EUA) e a seguir na Inglaterra (KOZASA, 2006; GOLEMAN, 1999), chegando ao Brasil na década seguinte, na esteira dos movimentos de contracultura, âmbito no qual esteve associada a práticas místicas e à busca de espiritualidade (ALMEIDA; NAVES, 2008). O contato do médico Robert Keith Wallace com a Science of Creative Intelligence (SCI), a ciência da consciência preconizada por Maharishi Mahesh Yogi ${ }^{2}$,

\footnotetext{
${ }^{1} \mathrm{O}$ presente trabalho não contou com apoio financeiro de nenhuma natureza para sua realização.

${ }^{2}$ Maharishi desenvolveu a técnica de meditação transcendental, a mais considerada em pesquisas acadêmicas. Tornou-se mundialmente conhecido como guru dos Beatles e dos Beach Boys, entre outras personalidades. Seu engajamento acadêmico gerou a fundação da Maharishi University of Management (MUM) em 1973, tendo o dr. Wallace como seu primeiro presidente. Disponível em https://www.mum.edu/about-mum/history. Acesso em: 5 abril 2017.
} 
motivou-o a realizar a primeira pesquisa sobre o tema com controle dos parâmetros fisiológicos, apresentada na Universidade da Califórnia em 1970 como tese de doutorado, intitulada Os efeitos fisiológicos da meditação transcendental: uma proposição do quarto maior estado de consciência. Esta pesquisa sugeriu a existência de um novo estado de consciência, responsável por alterações como aumento da resistência galvânica da pele, aumento da intensidade de ondas alfa lentas e atividade ocasional de ondas teta, o que veio a demarcar o campo da Fisiologia da Meditação (WALLACE; BENSON, 1972; KOZASA, 2006).

A abordagem científica dessas pesquisas clínicas na década de 1970 contribuiu para homologar a meditação como prática secular. Racionalizada, difundiu-se pela cultura de massa, em programas de TV, música pop e livros ${ }^{3}$. Atualmente, as pesquisas em meditação ampliaram seu escopo: é tratada como forma de desenvolvimento profissional (CHADE-MENG TAN, 2014), como prática de promoção da saúde, terapêutica complementar em doenças crônicas e dependências químicas e também na busca de performance no esporte (KOZASA, 2006). As diferentes abordagens clínicas que se sedimentaram para a pesquisa da meditação são duas: neurofisiológicas e a psicologia cognitivo-comportamental (MENEZES; DELL'AGLIO, 2009a; MENEZES; DELL'AGLIO, 2009b).

As abordagens neurofisiológicas, derivadas dos estudos supracitados de Wallace, sustentam a ideia de que as mudanças na atividade neuroelétrica podem interferir em mudanças fisiológicas (WALLACE et al.; 1971), Tese corroborada por Lazar et al. (2005), que apresentam resultados de mudança na neuroplasticidade a partir das observações destas alterações induzidas pelas práticas meditativas, o que vem aprofundando os conhecimentos sobre a estruturação cerebral. Atualmente, é relevante a ênfase nos estudos neurofisiológicos instrumentalizados por exames de imagens e testes fisiológicos resultando em diminuição de níveis de lactato sanguíneo, de cortisol salivar e da frequência cardíaca do praticante, bem como redução do consumo de oxigênio e aumento da eliminação de gás carbônico, indicando uma diminuição metabólica (MENEZES; DELL'AGLIO, 2009a; MAURA et al. 2006; TRAVIS; WALLACE, 1999; LAURE-GORE; MARSHALL, 2016).

A partir da abordagem cognitivo-comportamental, Slagter et al. (2007) sugerem que as práticas meditativas consistem em um treinamento subjetivo, com o fim de educar a mente, partindo da premissa comum entre a psicologia cognitiva e a meditação de que a interpretação dos fatos tem maior importância do que os fatos propriamente ditos. Danucalov e Simões (2006) evidenciam que as práticas meditativas produzem um estado de consciência particular, diferente das tradicionalmente conhecidas, como vigília, sono e sonho. Goleman (1999) afirma que a prática meditativa consiste em um relaxamento corporal simultâneo à atividade mental, cuja resposta se dá por mudanças refletidas no sistema nervoso central e autônomo (AFTANAS; GOLOCHEIKINE, 2001). O hipometabolismo basal, associado ao estado de alerta mental, proporciona a capacidade de modular funções fisiológicas involuntárias, tornando a meditação um eficiente método de biofeedback (MENEZES; DELL'AGLIO, 2009a; CAHN; POLICH, 2006).

Ainda que haja diferentes abordagens, no âmbito acadêmico a investigação de tais práticas parte de uma característica comum: o controle da atenção (GOLEMAN, 1999; CAHN; POLICH, 2006). Entre as funções cognitivas, a atenção é a mais evidenciada nos efeitos da meditação. Lutz et al. (2004) verificaram que meditadores budistas experientes apresentam níveis de concentração significativamente maiores quando comparados a um grupo controle. Brefczynski-Lewis et al. (2007) também perceberam que os praticantes apresentaram menor ativação das regiões cerebrais, sugerindo, assim, que a prática pode agir sobre a distribuição

\footnotetext{
${ }^{3} \mathrm{O}$ livro Transcendental meditation: discovering inner energy and overcoming stress, apresentado por Buckminster Fuller (premiado arquiteto e presidente da Mensa International - sociedade para pessoas de alto QI) ficou seis meses entre os best sellers de 1975, categoria não ficção, segundo a revista Time. Disponível em
} <https://content.time.com/time/magazine/article/0,9171,913711,00.html.>. Acesso em: 1 abr. 2017. 
das atividades cognitivas. O trabalho de Khalsa (2010) aponta uma alteração no eletroencefalograma (EEG) após uma intervenção de oito semanas com a prática meditativa, atentando a um aumento da ativação pré-frontal. Para Menezes e Dell'aglio (2009a), o treino da meditação pode modular mecanismos de atenção e potencializar a capacidade da memória de trabalho.

De acordo com Cahn e Polich (2006), a partir do direcionamento da atenção, pode-se classificar a diversidade das técnicas meditativas ${ }^{4}$ em dois estilos básicos: mindfulness e concentrativa. Alguns autores ainda sugerem um terceiro tipo, a meditação contemplativa, que consiste na integração das duas técnicas supracitadas (SHAPIRO et al. 2005).

O tipo mindfulness é caracterizado pela atenção plena dos sentimentos, pensamentos, sensações e fatores externos (como ambientais e sociais), existindo apenas a observação livre, sem análise ou julgamento (MENEZES; DELL'AGLIO, 2009b). A técnica foi ocidentalizada por Jon Kabat-Zinn em 1990, médico norte-americano com experiências meditativas na medicina clínica e fundador do programa de redução de estresse baseado em mindfulness.

Sobre a técnica concentrativa, Menezes e Dell'Aglio (2009b) caracterizam-na pela convergência da atenção em um objeto único, ignorando qualquer estímulo externo a ele. Desta maneira, essa prática direciona a atenção em uma atividade cerebral ou sensorial específica: respiração, repetição de um som ou imagem (CAHN; POLICH, 2006).

Diante da diversidade de técnicas, Cardoso et al. (2008) desenvolveram uma conceituação operacional, caracterizando a meditação a partir de cinco elementos, a saber: uso de uma técnica específica (claramente definida); relaxamento muscular em algum ponto do processo (como indicador do relaxamento psicofísico); relaxamento da lógica (não se envolver em sequências de pensamentos); um estado autoinduzido, e um artifício de autofocalização (âncora).

No âmbito esportivo, o alto rendimento compreende as atividades fundamentadas em níveis elevados de competitividade, o que demanda uma estrutura institucional diferenciada em relação a outras manifestações esportivas ${ }^{5}$. Pela exigência de alta dedicação, o atleta neste nível geralmente conta com qualquer forma de renda alternativa (contrato com entidades esportivas elou formas de patrocínio), que possibilitam a permanência em regime de dedicação compatível com o rendimento esperado (COSTA, 2007; BUENO, 2008).

Partindo desse exposto, a eficácia do alto rendimento requer uma interação de cinco capacidades: físicas, técnicas, táticas, biotipológicas e socioambientais (PAULO; FORJAZ, 2001; SISTO; GRECO, 1994). A efetividade da interação entre esses fatores depende de uma ativação funcional do intelecto cognitivo (SISTO; GRECO, 1994). Para o aprimoramento do desempenho esportivo do atleta, este é submetido a altas cargas de trabalho, que modulam o estresse e estimulam as adaptações biológicas necessárias (MATOS et al., 2014). Como os resultados apresentados em pesquisas sobre a meditação contribuem para uma melhor adaptação ao estresse (MENEZES; DELL'AGLIO, 2009a) e mostram resultados diretamente relacionados às capacidades que alicerçam o rendimento esportivo (tanto fisiológicas como cognitivas), fica evidente o papel da meditação cognitiva nas reações emocionais, comportamentais e nos parâmetros fisiológicos. Considerando os princípios da terapia cognitiva (BECK; AL-

\footnotetext{
${ }^{4}$ Lutz et al. (2007) sugerem uma grande variedade de técnicas para se atingir o processo meditativo. Goleman (1998) enumera 11 técnicas mais difundidas: bhakti hindu, cabala judaica, hesicasma cristão, sufismo, meditação transcendental, yoga ashtanga, tantra indiano, budismo tibetano, budismo zen, quarta via de Gurdjieff e consciência sem escolha de Krishnamurti.

$5 \mathrm{O}$ esporte de alto rendimento é assim entendido pela sua diferenciação do esporte de lazer e também do esporte educacional, sobretudo pelos aspectos de rigor técnico, racionalidade científica, alta competitividade, regulamentação de sua prática, organização internacional, corpo de dirigentes, aspectos midiáticos e mercadológicos e pelo devir profissional, conforme o Manifeste sur le Sport, publicado em 1964 pelo Conseil Internationale d'Éducation Physique et Sport, vinculado à UNESCO. Tal manifesto é considerado pioneiro sobre o tema nas políticas do esporte, com uma análise crítica de seus níveis e distinção dos seus objetivos (BUENO, 2008; TUBINO, 2010).
} 
FORD, 2000; KNAPP; BECK, 2008) de que a forma perceber e interpretar o ambiente (interno ou externo) influencia o jeito de se posicionar e agir no momento, e que a função de atribuir significado é controlar os vários sistemas psicológicos (por exemplo, comportamental, memória e atencional), pode-se inferir que, à medida que a consciência do emprego das diferentes estratégias cognitivas é ampliada, melhores serão a adaptação e a sensação de bem-estar biopsicossocial.

Nesta perspectiva, o presente estudo vem realizar uma revisão sistematizada da literatura sobre as pesquisas clínicas que associam a meditação ao esporte de alto rendimento. A partir desta perspectiva do esporte, pretende-se avaliar a efetividade dos resultados apresentados pelas pesquisas clínicas que tratam da meditação aplicadas a atletas, em relação a três variáveis: os protocolos utilizados (técnicas meditativas); as modalidades esportivas; os parâmetros analisados (neurofisiológicos ou comportamentais) e seus efeitos. Acredita-se que, ao final desta pesquisa, possamos esclarecer a influência de tais variáveis nos parâmetros do esporte de alto rendimento, de modo a subsidiar futuras pesquisas aplicadas ao campo do treinamento esportivo.

\section{Métodos e materiais}

Este trabalho ${ }^{6}$ consiste em uma revisão sistematizada de literatura (GALVÃO; PEREIRA, 2014), com método integrativo de tratamento de dados. A revisão integrativa da literatura sintetiza resultados anteriormente pesquisados e mostra, sobretudo, as conclusões do conjunto de referências científicas sobre um certo fenômeno. Consiste, pois, numa ampla visão do estado da arte do fenômeno analisado, com o propósito de produzir uma visão sintética e ampla de certo fenômeno, utilizando-se de uma variabilidade de amostras e uma multiplicidade de finalidades: "Dentre os métodos de revisão, a revisão integrativa é a mais ampla, sendo uma vantagem, pois permite a inclusão simultânea de pesquisa experimental e quase-experimental" (MENDES; SILVEIRA; GALVÃO, 2008, p. 760). Para Crossetti (2012), a revisão integrativa deveria responder às seguintes questões para avaliar o estado da arte de um fenômeno: "O que é conhecido? Qual é a qualidade do que é conhecido? O que deve ser conhecido? Qual é o próximo passo para investigação ou prática?” (p.8). Embora não haja consenso entre os autores sobre os procedimentos metodológicos adotados, o estudo crítico de Ganong (1987) ainda é referenciado atualmente e delineia as cinco principais etapas do método: definição das questões ou hipóteses; estabelecimento da amostragem; análise e categorização dos estudos; avaliação e interpretação dos resultados; síntese e apresentação da revisão. Tomando estas etapas como guia e seguindo a clareza dos procedimentos de Mata et. al (2011), apresentaremos a seguir as perguntas que norteiam a presente revisão, que serão retomadas na discussão dos resultados:

a) Quais são os protocolos utilizados para o treino da meditação em estudos com atletas de alto nível?

b) Que tipos de modalidades esportivas predominam e como se diferenciam os treinamentos com práticas meditativas nestes estudos?

c) Quais abordagens são utilizadas, quais parâmetros são mensurados e quais são os efeitos da meditação enunciados nestes estudos com atletas?

A partir da definição das perguntas, foi estabelecida a amostragem que compõe a revisão, num processo de pesquisa por palavras-chave que seguiu um vocabulário controlado: os termos "meditação e esporte de alto rendimento", "meditação e atleta" foram combinados

\footnotetext{
${ }^{6}$ O projeto foi submetido e aprovado pelo Comitê de Ética em Pesquisa da Universidade Federal de São Paulo (UNIFESP), CEP/UNIFESP n¹147/2016 (número do parecer: 1.705.231).
} 
com os desfechos de interesse, como sugerido por Sin et al. (2003). Os critérios de inclusão foram:

- artigos disponíveis nas quatro bases de dados: PubMed, Scientific Eletronic Library on Line (SCIELO), Literatura Latino-Americana e do Caribe em Ciências da Saúde (LILACS), Medical Literature Analysis and Retrieval System Online (MEDLINE);

- artigos em português, espanhol e inglês, com resumos disponíveis nas referidas bases publicados entre $1^{\circ}$ de janeiro de 1990 e 31 de dezembro de 2016 (incluindo os que estão disponíveis on-line do ano de 2016 e que podem ser publicados em 2017);

-artigos que apresentaram delineamento experimental, quase experimental e/ou observacional $^{7}$, realizados em humanos que tiveram como escopo a análise dos efeitos das práticas meditativas sobre os marcadores fisiológicos, psicológicos, psicossociais e biomecânicos.

Foram excluídos os seguintes estudos:

- artigos que realizaram intervenções em indivíduos não atletas, com fatores de risco, crianças, idosos e pessoas com déficit cognitivo;

- artigos caracterizados por revisão de literatura ou estudos de caso.

Para o refinamento da revisão, as pesquisas selecionadas foram submetidas a uma avaliação segundo parâmetros adaptados da escala PEDro para análise de qualidade metodológica, desenvolvida pela Physiotherapy Evidence Database ${ }^{8}$, com o intuito de ser aplicada em estudos experimentais. A escala tem uma pontuação com teto de dez itens, sendo aplicado um (1) ponto quando há existência de indicadores de evidências qualificadas e zero (0) quando há inexistência deles (SAMPAIO; MANCINI, 2007). Apesar de este instrumental sugerir que os critérios de validade e reprodutibilidade exigem a realização concomitante de duas pessoas, tal procedimento não foi adotado nesta revisão ${ }^{9}$. Para mais, foi analisado o tipo de intervenção a que os grupos foram submetidos, os protocolos para avaliação dos desfechos e os resultados apontados pelos autores.

Dos 1.097 trabalhos triados pelas palavras-chave, identificou-se pelo título/temática que a maior parte deles não abordava a relação da meditação com o esporte. Muitos dos trabalhos tratavam de outras práticas corporais, como chi gong e ioga, ou tinham fins terapêuticos. Feita essa triagem, foram eleitos 28 artigos, dos quais 12 foram excluídos pela leitura do resumo porque não abordaram estudos clínicos a partir de um protocolo de práticas meditativas. Por fim, após a leitura completa dos 16 trabalhos, três deles apresentaram uma metodologia qualitativa ou incluíram no estudo um público diferente do composto por atletas. Devido a isso, foram excluídos desta pesquisa, como mostrado na Figura 1.

\footnotetext{
${ }^{7}$ Seguindo os procedimentos da Revisão Integrativa, adotou-se o critério da variedade de perspectivas.

${ }^{8}$ Disponível em https://www.pedro.org.au/portuguese/downloads/pedro-scale/. Acesso em: 19 abr. 2017.

${ }^{9}$ A escala PEDro é derivada do método Delphi, um processo que se utiliza de uma série contínua de formulários para produzir informação com o objetivo de encontrar um consenso a partir de opiniões entre especialistas (SANTOS; AMARAL, 2004). Segundo Sampaio e Mancini (2007, p. 87), "a escala PEDro apresenta níveis moderados de confiabilidade entre avaliadores (ICC $=0,68$; IC 95\% $=0,57-0,76)$ ". A escala PEDro é uma ferramenta que busca um consenso na qualificação das evidências de um trabalho, portanto, não mede a validade das conclusões de um estudo e tampouco o nível de pontuação de um artigo evidencia que o tratamento tenha eficiência clínica. No presente estudo, a adaptação da escala PEDro foi utilizada pela capacidade de este instrumental evidenciar os fundamentos metodológicos dos artigos analisados e possibilitar uma leitura mais sistematizada e consistente das informações apresentadas.
} 
Figura 1. Diagrama da triagem e inclusão de trabalhos ${ }^{10}$

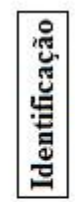

Foram encontrados 1097 artigos nas bases Medline, PubMed, Lilacs e Scielo.

Desses, 73 foram excluídos por duplicatas.
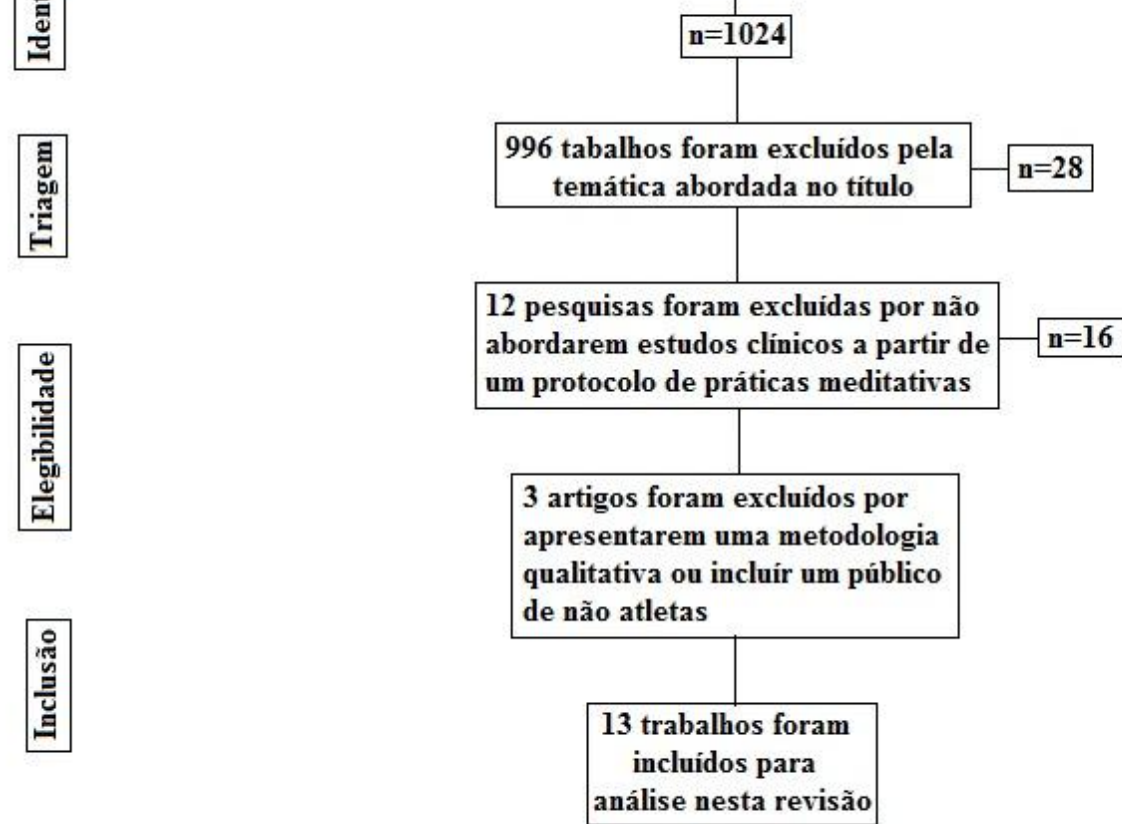

Tabela 1. Qualificação das Evidências

\begin{tabular}{ccc}
\hline Trabalho & País de Publicação & Qualificação das Evidências $^{*}$ \\
\hline Sekhon (2013) & Índia & $10 / 10$ \\
Moghadam et al (2013) & Bangladesh & $10 / 10$ \\
Maman P., Kanupriya G. (2012) & Índia & $10 / 10$ \\
John et al (2011) & Índia & $10 / 10$ \\
Bara Filho et al. (2002) & Brasil & $10 / 10$ \\
Hamilton, Schutte (2016) & Austrália & $10 / 10$ \\
Sekhon (2013) & Índia & $10 / 10$ \\
Mikicin, Marek (2015) & Estados Unidos & $8 / 10$ \\
Solberg et al (1996) & Noruega & $8 / 10$ \\
Rushenhack, et al (2016) & Costa Rica & $8 / 10$ \\
Ritch, et al (2015) & Estados Unidos & $8 / 10$ \\
Kaufman et al (2009) & Estados Unidos & $6 / 10$ \\
Bernier,. et al (2009) & França & $6 / 10$ \\
\hline
\end{tabular}

${ }^{10}$ Critérios: (1) especificação dos critérios de inclusão (item não pontuado); (2) alocação aleatória; (3) sigilo na alocação; (4) similaridade dos grupos na fase inicial ou basal; (5) mascaramento dos sujeitos; (6) mascaramento do terapeuta; (7) mascaramento do avaliador; (8) medida de pelo menos um desfecho primário em $85 \%$ dos sujeitos alocados; (9) análise da intenção de tratar; (10) comparação entre grupos de pelo menos um desfecho primário; (11) relato de medidas de variabilidade e estimativa dos parâmetros de pelo menos uma variável primária 
Sobre a qualificação das evidências a partir do questionário PEDro, sete pesquisas atingiram a pontuação máxima da escala (Sekhon, 2013a; Sekhon, 2013b; Moghadam et al., 2013; Maman P.; Kanupriya G., 2012; John et al., 2011; Bara Filho et al., 2002; Hamilton; Schutte, 2016); quatro não responderam aos critérios de alocação aleatória dos grupos estudados e de sigilo (Mikicin; Marek, 2015; Solberg et al.,1996; Rushenhack et al., 2016; Ritch et al., 2015), atingindo uma pontuação oito; e, nos trabalhos de Kaufman et al. (2009) e Bernier et al. (2009), não foram evidenciados os critérios referentes à alocação dos grupos estudados, ao mascaramento dos indivíduos e à análise da intenção, apresentando apenas seis pontos de 10 possíveis. As análises são apresentadas pela ordem decrescente de pontuação, iniciando pelos melhores qualificados na Tabela 1.

Optou-se pela apresentação dos resultados sintetizados em dois quadros (Tabela 2: Protocolos e abordagens e Tabela 3: Modalidades, parâmetros e efeitos da meditação), que foram integrados à análise e à discussão a partir das três perguntas que norteiam esta revisão integrativa, comentadas nos respectivos tópicos: a descrição dos protocolos utilizados para o treino da meditação em estudos com atletas de alto nível; as abordagens teóricas, parâmetros avaliados e efeitos da meditação nos atletas; e a predominância das modalidades esportivas e diferenciação dos treinos de práticas meditativas.

\section{Resultados e discussão}

Descrição dos protocolos utilizados para o treino da meditação em estudos com atletas de alto nível

Pela comparação entre as Tabelas 1 e 2 , notou-se que, embora a maioria dos estudos tenha sido publicada há menos de cinco anos (2013 em diante), a maior parte dos trabalhos melhor avaliados pela escala PEDro (8/10 e 10/10) utilizou técnicas desenvolvidas há mais de cinco décadas. Por outro lado, as duas pesquisas que apresentaram menor pontuação nesta mesma avaliação utilizaram protocolos criados há uma década. Tais evidências são um indicativo da preferência pelas técnicas clássicas nos estudos com maior rigor e controle sobre as variáveis que qualificam a pesquisa clínica, preferência essa associada ao país que se destaca na produção de pesquisas com a temática da meditação, a Índia, embora o interesse esportivo não esteja à altura. O protocolo de Mindfulness foi o mais recorrente, o que corrobora as análises de Bishop et al. (2004), que destacam os dois protocolos mais adaptados em pesquisas clínicas e científicas nos últimos 20 anos: Mindfulness Based Stress Reduction (MBSR), desenvolvido por Jon Kabat-Zinn (Universidade de Massachusetts), e Mindfulness Based Cognitive Therapy (MBCT), desenvolvido por uma equipe de psicólogos formada por John D. Teasdale e Mark Williams (Universidade de Oxford), além de Zindel Segal (Universidade de Toronto).

Além da clara definição da técnica utilizada, os estudos evidenciaram que os protocolos meditativos realizam procedimentos de relaxamento muscular e da lógica, assim como fizeram uso de âncora (artifício de autofocalização) na respiração ou em grupamentos musculares, atendendo ao conceito operacional de Cardoso et al. (2008). No entanto, apenas no estudo de Kaufman et al. (2009) foi observado um esforço de adaptação do protocolo meditativo às peculiaridades da modalidade esportiva, o que condiz com o princípio da especificidade do treinamento, que preconiza a especificidade da ação muscular, das fontes energéticas, qualidades físicas e coordenações psicomotoras na prescrição de um treino esportivo (MATVEEV, 1980; VERKHOSHANSHI, 2001). No entanto, deve-se ressaltar que o estar presente, aspecto central no mindfulness (GERMER SIEGEL; FULTON, 2016), constitui uma importante estratégia de controle da ansiedade, uma vez que o fluxo de pensamento do atleta não se volta para 
experiências passadas não positivas ou para as antecipações catastróficas do futuro. Portanto, estar focado no presente e na tarefa aumenta a probabilidade de sua execução com êxito.

Ainda sobre as relações entre protocolo e treinamento, notou-se que $70 \%$ dos estudos $(9 / 12)^{11}$ apresentaram um período de realização das práticas meditativas entre quatro e nove semanas (Tabela 2), uma predominância de periodicidade diária (6/12) e duração da sessão de até 30 minutos (7/12). No entanto, o tempo total de horas semanais de treino meditativo não variou substancialmente na maioria dos estudos (9/12), com variações entre 90 e 150 minutos, pois os protocolos com periodicidade de prática semanal (uma ou duas vezes por semana) apresentaram maiores durações das sessões, entre 90 e 150 minutos. Segundo os modelos modernos de periodização do treinamento (DIAS et al., 2016), há uma variação ideal de dois a oito meses para se atingir o pico de performance do atleta, ou seja, as intervenções meditativas encontradas corresponderam a cerca de $5 \%$ do planejamento esportivo ideal. Esses dados sugerem que os efeitos positivos da meditação podem ser observados em um período curto de prática. Contudo, nenhum estudo apresentou dados sobre a realização da meditação dentro de continuado de periodização esportiva. 
Tabela 2. Protocolos e Abordagens

\begin{tabular}{|c|c|c|c|}
\hline Trabalhos & Técnicas & Origem & Descrição \\
\hline Sekhon (2013) & $\begin{array}{l}\text { Meditação transcendental/ } \\
\text { relaxamento muscular } \\
\text { progressivo }\end{array}$ & $\begin{array}{l}\text { Maharishi Mahesh Yogi } \\
\text { (1958) / Edmund } \\
\text { Jacobson (1920) }\end{array}$ & $\begin{array}{l}\text { Duração: } 6 \text { semanas com duas sessões } \\
\text { diárias de } 25 \text { minutos } \\
\text { Objetivo: - /Estimular a consciência do } \\
\text { praticante em contrair e relaxar todos os } \\
\text { grupamentos musculares }\end{array}$ \\
\hline Moghadam et al (2013) & Técnicas de mindfulness & $\begin{array}{l}\text { Protocolo desenvolvido } \\
\text { pelos próprios } \\
\text { pesquisadores }\end{array}$ & $\begin{array}{l}\text { Duração: - } \\
\text { Objetivo: Estimular a "consciência } \\
\text { universal"/ "consciência em massa" }\end{array}$ \\
\hline $\begin{array}{c}\text { Maman P., Kanupriya G. } \\
\text { (2012) }\end{array}$ & Biofeedback & $\begin{array}{l}\text { Sigmund Jacobson } \\
\text { (1938) }\end{array}$ & $\begin{array}{l}\text { Duração: } 10 \text { dias consecutivos com } \\
\text { sessões de } 20 \text { minutos }\end{array}$ \\
\hline & & & $\begin{array}{c}\text { Objetivo: autorregulação dos parâmetros } \\
\text { fisiológicos através de aparelhos } \\
\text { eletrônicos }\end{array}$ \\
\hline John et al (2011) & $\begin{array}{l}\text { Mindfulness meditation } \\
\text { therapy }\end{array}$ & $\begin{array}{l}\text { Protocolo desenvolvido } \\
\text { pelos próprios } \\
\text { pesquisadores }\end{array}$ & $\begin{array}{l}\text { Duração: } 4 \text { Semanas com } 6 \text { sessões de } \\
\qquad 20 \text { minutos } \\
\text { Objetivo: Completar } 5 \text { ciclos } \\
\text { respiratórios por minuto, utilizando um } \\
\text { metrônomo }\end{array}$ \\
\hline Bara Filho et al. (2002) & $\begin{array}{l}\text { Relaxamento Muscular } \\
\text { progressivo }\end{array}$ & $\begin{array}{l}\text { Sigmund Jacobson } \\
\text { (1920) }\end{array}$ & $\begin{array}{l}\text { Duração: } 7 \text { semanas com duas sessões } \\
\text { semanais de } 20-30 \text { minutos } \\
\text { Objetivo: estimular a consciência do } \\
\text { praticante em contrair e relaxar todos os } \\
\text { grupamentos musculares }\end{array}$ \\
\hline Hamilton, Schutte (2016) & $\begin{array}{c}\text { Mindfulness-integrated } \\
\text { Cognitive behavior Therapy } \\
\text { (adaptação) }\end{array}$ & - & $\begin{array}{l}\text { Duração: } 8 \text { Semanas com encontros de } \\
120 \text { minutos semanais } \\
\text { Objetivo: Estimular a plena atenção nos } \\
\text { processos cognitivos e pensamentos }\end{array}$ \\
\hline Sekhon (2013) & $\begin{array}{c}\text { Meditação } \\
\text { transcendental/relaxamento } \\
\text { muscular progressivo }\end{array}$ & $\begin{array}{l}\text { Maharishi Mahesh Yogi } \\
\text { (1958) / Edmund } \\
\text { Jacobson (1920) }\end{array}$ & $\begin{array}{l}\text { Duração: } 6 \text { semanas com duas sessões } \\
\text { diárias de } 25 \text { minutos } \\
\text { Objetivo: - /estimular a consciência do } \\
\text { praticante em contrair e relaxar todos os } \\
\text { grupamentos musculares }\end{array}$ \\
\hline Mikicin, Marek (2015) & $\begin{array}{c}\text { Relaxamento } \\
\text { audiovisual/Treinamento } \\
\text { autógeno de Schultz }\end{array}$ & $\begin{array}{l}\text { Protocolo desenvolvido } \\
\text { pelos próprios } \\
\text { pesquisadores /Johannes } \\
\text { Heinrich Schultz (1930) }\end{array}$ & $\begin{array}{l}\text { Duração: } 7 \text { meses como sessões diárias } \\
\text { de } 45 \text { minutos } \\
\text { Objetivo: induzir o relaxamento através } \\
\text { de imagens e sons/ autossugestão para } \\
\text { atingir determinado estado de } \\
\text { concentração }\end{array}$ \\
\hline Solberg et al (1996) & Meditação ACEM & Are Holan (1966) & $\begin{array}{c}\text { Duração: } 7 \text { Semanas com práticas } \\
\text { diárias de } 30 \text { minutos }\end{array}$ \\
\hline & & & $\begin{array}{c}\text { Objetivo: Praticar o não julgamento e a } \\
\text { livre observação dos fatos internos e } \\
\text { externos. }\end{array}$ \\
\hline
\end{tabular}




\begin{tabular}{|c|c|c|c|}
\hline Rushenhack, et al (2016) & $\begin{array}{l}\text { Mindfulness Acceptance } \\
\text { Commitment }\end{array}$ & $\begin{array}{l}\text { Frank L. Gardner e Zella } \\
\text { E. Moore }(2004)\end{array}$ & $\begin{array}{l}\text { Duração: } 9 \text { semanas como sessões } \\
\text { semanais de } 90 \text { minutos } \\
\text { Objetivo: Proporcionar a experiência de } \\
\text { flow nos atletas, fazendo-os desenvolver } \\
\text { a habilidade de compreender as } \\
\text { sensações internas (pensamentos, } \\
\text { emoções) e corporais como algo } \\
\text { transitório à vivência humana. }\end{array}$ \\
\hline Ritch, et al (2015) & $\begin{array}{c}\text { Técnicas de respiração } \\
\text { abdominal e mentalização da } \\
\text { respiração }\end{array}$ & $\begin{array}{l}\text { Protocolo desenvolvido } \\
\text { pelos próprios } \\
\text { pesquisadores }\end{array}$ & $\begin{array}{l}\text { Duração: } 8 \text { semanas com práticas diárias } \\
\qquad \text { de } 20-30 \text { minutos } \\
\text { Objetivo: Meditação aos exercícios } \\
\text { respiratórios associados à posturas e } \\
\text { movimentos corporais. }\end{array}$ \\
\hline Kaufman et al. (2009) & $\begin{array}{c}\text { Mindfulness Sport } \\
\text { Performance Enhancement } \\
\text { (MSPE) }\end{array}$ & $\begin{array}{l}\text { Keith A. Kaufman e } \\
\text { Carol R. Glass (2006) }\end{array}$ & $\begin{array}{l}\text { Duração: } 6 \text { Semanas com encontros de } \\
90 \text { minutos semanais } \\
\text { Objetivo: Tornar a prática guiada de } \\
\text { mindfulness uma vivência, incluindo } \\
\text { exercícios específicos da modalidade } \\
\text { esportiva }\end{array}$ \\
\hline Bernier,. et al. (2009) & $\begin{array}{l}\text { Mindfulness based } \\
\text { Cognitive Therapy } \\
\text { (Adaptação) }\end{array}$ & $\begin{array}{l}\text { Jhon D. Teasdale/ Zindel } \\
\text { Segal e J. Mark G. } \\
\text { William (2002) }\end{array}$ & $\begin{array}{l}\text { Duração: } 8 \text { Semanas com encontros de } \\
\qquad \text { hh semanais } \\
\text { Objetivo: Estimular a plena atenção nos } \\
\text { processos cognitivos e pensamentos }\end{array}$ \\
\hline
\end{tabular}

\section{Abordagens teóricas, parâmetros avaliados e efeitos da meditação nos atletas}

Os estudos clínicos encontrados têm predominância da abordagem cognitivocomportamental da meditação (70\% dos estudos), em relação àqueles de abordagem neurofisiológica (30\% dos estudos). Apenas o estudo de Mikicin (2015) combinou parâmetros neurofisiológicos e comportamentais (EEG banda alfa e testes cognitivos), podendo ser caracterizado como multidisciplinar.

Os trabalhos de Bara Filho et al. (2002), John, S. et al. (2011) e Mikicin e Marek (2015) avaliaram mudanças da atividade cerebral por EEG e valores plasmáticos e sanguíneos de cortisol, respectivamente. Bara Filho et al. (2002) afirmam que o aumento nos níveis de estresse ativa o sistema endócrino, resultando na maior liberação de hormônios glicocorticoides, tal como o cortisol. Altas quantidades desse hormônio geram situações de perda de controle, depressão e, principalmente, distresse. Os mesmos autores sugerem que práticas de relaxamento estimulam uma homeostasia na atividade do sistema nervoso simpático e parassimpático, otimizando os efeitos da recuperação e a performance esportiva. Em ambos os trabalhos, houve redução significativa do cortisol, bem como uma alteração no EEG dos atletas que foram submetidos à intervenção. Tal mudança na ativação cerebral se mostrou evidente, principalmente no córtex pré-frontal, responsável por tomadas de decisão, igualmente observado em um exame feito após o processo de aprendizagem motora (LUFT; ANDRADE 2006). Os mesmos autores expõem a ideia de que o aumento da atividade alfa nas áreas prémotoras indica uma consolidação do gesto motor, a mesma mudança encontrada por Mikicin e Marek (2015) após a prática meditativa.

Grande parte dos delineamentos clínicos envolvendo a meditação no esporte preocupase em quantificar os processos cognitivos após a intervenção. Esse fato pode ser justificado por Gonçalves e Belo (2007), ao afirmarem que uma das limitações do desempenho esportivo 
são os processos psicobiológicos, com destaque à ansiedade. Entre artigos analisados, os parâmetros de avaliação preponderantes foram a performance e a ansiedade, tendo também destaque $\mathrm{o}$ aspecto da atenção.

Atletas com altos níveis de ansiedade podem encarar um evento competitivo importante como algo ameaçador, caracterizando um ambiente catabólico e suprimindo o aporte energético necessário para o alto rendimento. A revisão de Vanderbergh e Assunção (2009) embasa esta ideia e apresenta resultados favoráveis à redução de estresse, problemas psicossomáticos, dor crônica e transtornos de ansiedade.

Para mais, dois parâmetros analisados merecem destaque. São eles: pessimismo e aceitação. $\mathrm{O}$ primeiro diz respeito à visão do indivíduo sobre a realidade. Percepções negativas do ambiente ocasionam baixa motivação para a realização de tarefas, bem como aumento dos níveis de ansiedade (GONÇALVES; BELO, 2007). Não obstante, a aceitação remete ao estado de alento com a realidade, ou seja, a satisfação com o rendimento obtido, no caso.

Os resultados dessas análises mostraram que a intervenção meditativa reduziu os níveis de pessimismo nos atletas e aumentou a aceitação do grupo experimental. Essas alterações contribuíram para um superávit no desempenho esportivo de ambos.

A análise do flow presente nos estudos de Hamilton e Schutte (2016) e Kaufman et al. (2009) remetem à experiência de êxtase em realizar a tarefa esportiva. Neste parâmetro, os valores apresentados mostraram-se significativamente maiores no grupo experimental. Segundo Massarela e Winterstein (2009), a teoria do flow consiste em um estado de plena satisfação e felicidade com a tarefa realizada e, para isso, deve atingir um equilíbrio entre desafio e habilidade; metas objetivas e feedback dos resultados, e ação e consciência. A meditação pode ser utilizada como um meio de conhecimento dos nossos pensamentos e emoções, condicionando-nos a entendê-los e a gerar ações mais conscientes (KOZASA, 2007). Peres et al. (2007) sugerem que a capacidade de informações assimiladas pelo cérebro é reduzida se comparada com a quantidade com que são fornecidas. Expõem ainda que tais experiências são resultado de crenças e do histórico de vida, criando critérios de escolha e um limiar de observação. Portanto, a auto-observação induzida pela meditação rompe com estes critérios de escolha, flexibilizando o limiar de observação, consequentemente, proporcionando novas experiências e sensações, nas quais o flow está incluído.

Tabela 3. Modalidades, parâmetros e efeitos da meditação

\begin{tabular}{|c|c|c|c|}
\hline $\begin{array}{l}\text { Tra- } \\
\text { balhos }\end{array}$ & $\begin{array}{l}\text { Modalidade Es- } \\
\text { portiva }\end{array}$ & Parâmetros Avaliados & $\begin{array}{l}\text { Efeitos da Medita- } \\
\text { ção }\end{array}$ \\
\hline $\begin{array}{l}\text { Sekhon } \\
(2013)\end{array}$ & Tiro de rifle & Performance & $\begin{array}{l}\text { Melhora significa- } \\
\text { tiva de performance (G. } \\
\text { relaxamento muscular } \\
\text { progressivo) }\end{array}$ \\
\hline
\end{tabular}

$\begin{array}{lcc}\begin{array}{l}\text { Moghadam } \\ \text { et al (2013) }\end{array} & \text { Badminton } & \text { Performance e ansiedade } \\ \begin{array}{l}\text { Maman e } \\ \text { Kanupriya } \\ (2012)\end{array} & \text { Basquetebol } & \text { Ansiedade } \\ \begin{array}{l}\text { John et al } \\ (2011)\end{array} & \text { Tiro } & \begin{array}{r}\text { Performance/estresse pré- } \\ \text { competição/cortisol salivar }\end{array}\end{array}$

Bara Filho et al. (2002)
Natação

Cortisol sanguíneo

\section{Redução significa- tiva da ansiedade \\ Redução significa- tiva da ansiedade}

Redução significativa do cortisol salivar e do estresse pré-competição e aumento da performance

Redução significativa do cortisol sanguíneo 


\begin{tabular}{|c|c|c|c|}
\hline $\begin{array}{l}\text { Hamilton, } \\
\text { Schutte } \\
(2016)\end{array}$ & Ciclismo & Flow/ansiedade/pessimismo & $\begin{array}{l}\text { Aumento da experi- } \\
\text { ência de flow e redução } \\
\text { significativa da ansiedade }\end{array}$ \\
\hline $\begin{array}{l}\text { Sekhon } \\
(2013)\end{array}$ & Tiro de pistola & Performance & $\begin{array}{l}\text { Melhora significa- } \\
\text { tiva de performance ( } \mathrm{G} \text {. } \\
\text { relaxamento muscular } \\
\text { progressivo) }\end{array}$ \\
\hline $\begin{array}{l}\text { Mikicin, } \\
\text { Marek } \\
(2015)\end{array}$ & $\begin{array}{c}\text { Nata- } \\
\text { ção/esgrima/atletismo/ } \\
\text { taekwondo/judô }\end{array}$ & $\begin{array}{l}\text { EEG (banda alfa) e Testes } \\
\text { Cognitivos }\end{array}$ & $\begin{array}{l}\text { Aumento da capa- } \\
\text { cidade de relaxamento e } \\
\text { mudança a longo prazo no } \\
\text { EEG }\end{array}$ \\
\hline $\begin{array}{l}\text { Solberg et al } \\
(1996)\end{array}$ & Tiro & Performance e estresse & $\begin{array}{l}\text { Diminuição signifi- } \\
\text { cativa do estresse }\end{array}$ \\
\hline $\begin{array}{l}\text { Rushenhack, } \\
\text { et al (2016) }\end{array}$ & Voleibol & Atenção & $\begin{array}{l}\text { Aumento significa- } \\
\text { tivo da atenção }\end{array}$ \\
\hline $\begin{array}{l}\text { Ritch, et al } \\
(2015)\end{array}$ & Natação & Marcadores cardíacos & $\begin{array}{l}\text { Redução da fre- } \\
\text { quência cardíaca }\end{array}$ \\
\hline $\begin{array}{l}\text { Kaufman et } \\
\text { al. (2009) }\end{array}$ & $\begin{array}{l}\text { Tiro com arco e } \\
\text { golfe }\end{array}$ & $\begin{array}{c}\text { Ansieda- } \\
\text { de/perfeccionismo/confiança/ aten- } \\
\text { ção/flow }\end{array}$ & $\begin{array}{l}\text { Melhora significa- } \\
\text { tiva dos parâmetros avali- } \\
\text { ados }\end{array}$ \\
\hline $\begin{array}{l}\text { Bernier,. et } \\
\text { al. (2009) }\end{array}$ & Golfe & $\begin{array}{l}\text { Autoconfiança/compromisso/ } \\
\text { estresse/relaxamento e ativa- } \\
\text { ção/ planejamento }\end{array}$ & $\begin{array}{l}\text { Aumento significa- } \\
\text { tivo dos parâmetros }\end{array}$ \\
\hline
\end{tabular}

\section{A predominância das modalidades esportivas e diferenciação dos treinos de práticas meditativas}

Os resultados da Tabela 3 evidenciam uma quantidade maior de trabalhos realizados com modalidades esportivas individuais. Segundo Gonçalves e Belo (2007), em modalidades individuais, os atletas não dividem a responsabilidade, expondo-se sozinhos a uma avaliação direta dos espectadores, o que gera uma pressão maior de êxito no desempenho. Corroborando essa ideia, Zeng (2003) sugere que os atletas de modalidades coletivas têm menores níveis de ansiedade somática quando comparados com atletas de esportes individuais. Portanto, atletas de modalidades individuais estão mais susceptíveis a níveis de estresse maiores do que os de modalidades coletivas, além de uma percepção de ameaça elevada, resultando em decisões de estratégias menos eficazes (DIAS, 2005), fato que contribui para justificar os resultados encontrados, de menor incidência de pesquisas com intervenção meditativa em modalidades coletivas. Além disso, a dificuldade de guiar tais práticas amplia-se no trabalho em grupo, uma vez que a individualidade de seus membros pode gerar respostas distintas. O treinamento mental no âmbito esportivo apresenta igual importância ao condicionamento físico (SAMULSKI, 2011; WEINBERG; GOULD, 2001). No entanto, as demandas psicológicas, assim como as físicas, dependerão das características pessoais do atleta e do esporte.

Corroborando as ideias anteriores, independente da duração da modalidade esportiva em questão, níveis ótimos de atenção e concentração predizem o desempenho esportivo (JUNIOR et al., 2003), ressaltando a necessidade de adaptações destes parâmetros em diferentes situações. Sobre a atenção, pode ser caracterizada quanto a sua amplitude e a sua direção, sendo, respectivamente, ampla (vários estímulos) ou estreita (focada em poucos estímulos) e associativa (interna) ou dissociativa (externa) (NIDEFFER, 1978). Atletas de provas de longa dura- 
ção bem-sucedidos utilizam uma técnica mista entre a atenção associativa e dissociativa durante a execução da prova (WEINBERG; GOULD, 2001), ao passo que velocistas buscam a melhor concentração através da atenção associativa (SILVA, 2013). Porém, Junior et al. (2003) sugerem que, para eventos presentes em modalidades de curta duração, como o momento da largada, o tipo de atenção utilizada é dissociativa e estreita, uma vez que o atleta focaliza sua atenção em poucos estímulos externos (sinal da partida).

Segundo Samulski (2002), a flexibilização da atenção garantirá o rendimento esportivo ao atleta. Tal variância é corroborada pelo trabalho de Menezes et al. (2011), ao exporem que se supera a distração a partir de uma estabilidade mental, adquirida pelo treinamento meditativo mais focalizado (concentrativo/estreito) ou pela focalização mais ampla da atenção (mindfulness/amplo).

\title{
Considerações finais
}

Os estudos analisados nesta revisão mostram um interesse em crescimento na temática da meditação no esporte notadamente nos últimos cinco anos e estudos ainda escassos no Brasil. Os estudos revisados apresentam índices elevados de rigor metodológico e controle sobre as variáveis que qualificam a pesquisa observacional (escala PEDro). Entre os resultados apresentados, foi evidenciado o uso de técnicas clássicas de meditação, com destaque para protocolos adaptados de Mindfullness, em programas com duração entre 90 e 150 minutos semanais, periodicidade diária e sessões de até 30 minutos. Entre os protocolos utilizados, notou-se baixa evidência de organização da prática meditativa conforme fundamentos do treinamento esportivo, como especificidade, continuidade e periodização, o que pode ser indicativo da ausência de profissionais de Educação Física integrados em equipes multidisciplinares de pesquisadores, cujas abordagens seguem parâmetros predominantemente comportamentais e neurofisiológicos.

As modalidades individuais foram as mais presentes nesta revisão, o que evidencia não apenas uma maior capacidade de controle clínico, mas também uma maior susceptibilidade destes atletas a níveis de estresse e ansiedade do que aqueles de modalidades coletivas. Acredita-se que a percepção de um resultado oriundo exclusivamente do seu desempenho contribua para a abertura de novas estratégias que ampliem a sensação de segurança e controle. Ademais, os estudos analisados demonstraram impacto positivo da meditação nos preditores do desempenho esportivo, como os marcadores fisiológicos diretamente relacionados ao aprimoramento físico, às modulações cognitivas influentes nas condutas técnicas/táticas do esporte e às alterações socioambientais adquiridas pela sensibilidade de análise da situação momento. Ainda que não haja estudos conclusivos, há fortes indicativos de que futuros estudos clínicos com protocolos de meditação no esporte devem abranger maior diálogo com as teorias do treinamento e serem conduzidos por equipes multidisciplinares.

\section{THE MEDITATION IN HIGH PERFORMANCE SPORT: A INTEGRATIVE RE- VIEW}

\begin{abstract}
This article analyzes the evidence in observational studies that have evaluated the athletic performance of individuals in meditation programs. The research was carried out on scientific bases such as Lilacs, Scielo, Pubmed and Medline. The evidence was evaluated according to the PEDro scale, where the protocols used for training, sports modalities and meditation effects were systematized. We analyzed 13 studies that showed a positive correlation between meditation and increased athletic performance. Among them, behavioral approaches and the
\end{abstract}


evaluation of cognitive predictors of individual athletic performance predominated. Despite the positive outcomes, the used meditation protocols have demonstrated to be inconsistent according to the sports training fundamentals.

Keywords: Meditation. Athletic performance. Sport.

\section{LA MEDITACIÓN EN EL DEPORTE DE ALTO RENDIMIENTO: REVISIÓN IN- TEGRADORA DE LA LITERATURA}

\section{Resumen}

Este artículo analiza las evidencias de estudios de observación que evaluaron el desempeño deportivo de atletas bajo programas de meditación. La investigación se realizó en las bases: Lilacs, Scielo, PubMed y Medline. Se calificaron las evidencias por la escala PEDro y se tabularon los protocolos utilizados para el entrenamiento; los parámetros evaluados y efectos de meditación en la performance; y las modalidades deportivas. Se analizaron 13 trabajos que presentaron asociación positiva entre meditación y aumento de la performance, con predominio de abordajes de comportamiento y evaluación de los predictores cognitivos del rendimiento. A pesar de los resultados positivos, los protocolos de meditación demuestran inconsistencias con los fundamentos del entrenamiento desportivo.

Palabras clave: Meditación. Rendimiento Atlético. Deporte.

\section{Referências}

AFTANAS, L. I.; GOLOCHEIKINE, S. A. Human anterior and frontal midline theta and lower alpha reflect emotionally positive state and internalized attention: high-resolution EEG investigation of meditation. Neuroscience Letters, v. 310, p. 57-60, 2001.

ALMEIDA, M. I. M; NAVES, S. C. "Por quê não?": rupturas e continuidades da contracultura. Rio de Janeiro: Editora 7 letras, 2008.

BARA FILHO, M.G. et al. A redução dos níveis de cortisol sanguíneo através da técnica de relaxamento progressivo em nadadores. Revista Brasileira de Medicina e Esporte, v. 8, n. 4, p. 139-143, 2002.

BERNIER, M. et al. Mindfulness and acceptance approaches in sport performance. Journal of Clinical Sports Psychology, n. 4, p. 320-333, 2009.

BISHOP, S. R. et al. Mindfulness: a proposed pperational definition. Clinical Psychology: Science and Practice, n. 3, v.11, p. 230-241, 2004.

BREFCZYNSKI-LEWIS et al. Neural correlates of attentional expertise in long-term meditation practitioners. Proceedings of the National Academy of Sciences, v.104, n.27, p.1148311488, 2007.

BUENO, L. Políticas públicas do esporte no Brasil: razões para o predomínio do alto rendimento. 2008. 200f. Tese (Doutorado em Administração Pública e Governo) - Escola de Administração de Empresas de São Paulo da Fundação Getúlio Vergas, São Paulo, 2008.

CAHN, B.; R., POLICH, J. Meditation states and traits: EEG, ERP and neuroimaging studies. Psychological Bulletin. v.132, n.2, p.180-211, 2006. 
CORDOSO, L.;V. DORNELES, B. V. Qual o papel que a memória de trabalho exerce na aprendizagem da matemática? Bolema, v.26, n.42, p. 627-647, 2008.

CROSSETTI, M.G.O. Revisão integrativa de pesquisa na enfermagem - o rigor cientifico que lhe é exigido [editorial]. Revista Gaúcha de Enfermagem, v. 33, n. 2, p. 8-9, 2012.

DANUCALOV, M.; SIMÕES, R. Neurofisiologia da meditação. São Paulo: Phorte Editora, 2006.

DIAS, C. S. L.. Do stress e ansiedade às emoções no desporto: da importância da sua compreensão à necessidade da sua gestão. 2005. Tese (Doutorado) - Universidade do Minho, Braga, Portugal, 2005.

FIGUEIREDO, O. Estabelecendo pontes entre o Oriente e o Ocidente: um contributo da perspectiva budista na educação para a sustentabilidade. Revista Interacções, v. 3, p. 122-150, 2006.

GANONG, L. H. Integrative reviews of nursing research. Research in Nursing \& Health, v. 10, p. 1-11, 1987.

GERMER, C. K.; SIEGEL, R. D.; FULTON, R. R. Mindfulness e psicoterapia. Porto Alegre: Artmed, 2016.

GOLEMAN, D. Práticas de meditação. In: A arte da meditação: um guia para meditação. Rio de Janeiro: Sextante, 1999.

GONÇALVES, M. P.; BELO, R. P. Ansiedade-traço competitiva: diferenças quanto ao gênero, faixa etária, experiência em competições e modalidade esportiva em jovens atletas. Psicologia-USF, v. 12, n. 2, p. 301-307, 2007.

HAMILTON, J. S.; SHUTTE, N. S. Effects of a mindfulness intervention on sports anxiety, pessimism, and flow in competitive cyclists. Applied Psychology: Health and Well-Being, v. 8, n. 1, p. 85-103, 2016.

JOHN, S. et al. The effect of mindfulness meditation on HPA-Axis in pre-competition stress in sports performance of elite shooters. National Journal of Integrated Research in Medicine, v. 2, n. 3, p. 15-21, 2011.

JÚNIOR, G. V. P. et al. Concentração: aspecto psicológico relevante para o desempenho esportivo. Revista Mineira Educação Física, v. 11, n. 2, p. 42-74, 2003.

KAUFMAN, K. A. et al. Evaluation of mindful sport Performance enhancement (MSPE): a new approach to promote flow in athletes. Journal of Clinical Sports Psychology, v. 4, p. 334-356, 2009.

KOZASA, E. H. A prática de meditação aplicada ao contexto da saúde. Saúde Coletiva, v. 10, n. 3, p.63-66, 2006.

Medicina complementar e alternativa. Saúde Coletiva. v.28, n.6, p. 37-38, 2009. 
LAURES-GORE, J.; MARSHALL, R. Mindfulness meditation in aphasia: A case report. Journal of Neuro Rehabilitation, v. 38, n. 4, p. 321-329, 2016.

LAZAR, S. W. et al. Meditation experience is associated with increased cortical thickness. Neuroreport, v.16, n.17, p.1893-1897, 2005.

LEVINE, M. The positive psychology of buddhism and yoga: paths to a mature happiness. Lawrence Erlbaum Associates, v.13, n.2, 2000.

LUFT, C.; ANDRADE, A. A pesquisa com EEG aplicada à área de aprendizagem motora. Revista Portuguesa da Ciência Desportiva, v.6, n.1, p. 106-115, 2006.

LUTZ, A. et al. Long-term meditators self-induce high-amplitude gamma synchrony during mental practice. Proceedings of the National Academy of Sciences, v. 101, n. 46, p. 1636916373, 2004.

LUTZ, A. et al. Attention regulation and monitoring in meditation. Trends Cognitive Science Journal, v.12, n.4, p. 163-169, 2007.

MAHER, C.G. et al. Reliability of the PEDro scale for rating quality of randomized controlled trials. Physical Therapy, v.83, p.713-21, 2003.

MAMAN, P.; KANUPRIYA, G. The effect of heart rate variability biofeedback on performance psychology of basketball players. Psychophysiol Biofeedback, v.37, p. 131-144, 2012.

MASSARELA, F. L.; WINTERSTEIN, P. J. A motivação intrínseca e o estado mental flow em corredores de rua. Revista Movimento, v.15, n. 2, p. 45-68, 2009.

MATA, F.G. et al. Avaliação neuropsicológica do processo de tomada de decisões em crianças e adolescentes: uma revisão integrativa da literatura. Revista de Psiquiatria Clínica, v. 38, n. 3, p. 106-15, 2011.

MATOS, F.O. et al. High loads of training affect cognitive functions in soccer players. Revista Brasileira de Medicina e Esporte, v.20, n.5, p. 388-393, 2014.

MATVEEV, L. P. Fundamentos del entrenamiento desportivo. Espanha: MIR, 1980.

MAURA, P. et al. Effects of a randomized controlled trial of transcendental meditation on components of the metabolic syndrome in subjects with coronary heart disease. Archives of Internal Medicine, v. 166, n. 11, p. 1218-1224, 2006.

MENDES, K.D.S.; SILVEIRA, R.C.C.P; GALVÃO, C. M.. Revisão integrativa: método de pesquisa para a incorporação de evidências na saúde e na enfermagem. Texto \& Contexto Enfermagem, v. 17, n. 4, p. 758-764, 2008.

MENEZES, C. B. et al. Meditação, bem-estar e a ciência psicológica: revisão de estudos empíricos. Interação em Psicologia,v. 15, n.2, p. 239-248, 2011. 
MENEZES, C.B.; DELL'AGLIO, D.D. Os efeitos da meditação à luz da investigação científica em psicologia: revisão de literatura. Psicologia, ciência e profissão, v. 29, n. 2, p 276289, 2009a.

Por que meditar? A experiência subjetiva da prática de meditação. Psicologia em Estudo, v. 14, n. 3, p. 565-573, 2009 b.

MIKICIN, M .; MAREK, K. Audio-visual and autogenic relaxation alter amplitude of Alpha EEG band, causing improvements in mental work performance in athletes. Psychophysiol Biofeedback, v.40, p.219-227, 2015.

MOGHADAM, M. S. et al. Impact assessment of mindfulness techniques education on anxiety and sports performance in Badminton players Isfahan. International Research Journal of Applied and Basic Sciences, v.4, n. 5, p. 1170-1175, 2013.

NIDEFFER, R. M. The relationship of attention and anxiety to performance. In: STRAUB, W. F. (Ed.). Sport psychology: an analysis of athletic behavior. Ithaca, NY: Human Movement, 1978

ORSILLO, S. M.; ROEMER, L. Mindfulness-based stress reduction. In.: BRANTLEY, L. (Ed.) Acceptance and mindfulness-based approaches to anxiety, v.2, p. 131-145, 2005.

PAULO, A. C.; FORJAZ, C. L. M. Treinamento físico de endurance e força máxima: adaptações cardiovasculares e relações com a performance esportiva. Revista Brasileira de Ciências do Esporte, v. 22, n. 2, p. 99-114, 2001.

PERES, J. F. P et al. Espiritualidade, religiosidade e psicoterapia. Revista Psiquiatria Clínica, v. 34, n. 1, p. 136-145, 2007.

RITCH, T. A. et al. Assessment of cardiovascular parameters during meditation with mental targeting in varsity swimmers. Evidence-Based Complementary and Alternative Medicine, v.1, p. 1-5, 2016.

RUSHENHACK, M. C. et al. The effect of nine sessions of mindfulness-acceptancecommitment on sport performance and attention in volleyball players. American College of Sports Medicine, 2016.

SAMPAIO, R.F.; MANCINI, M.C. Estudos de revisão sistemática: um guia para síntese criteriosa da evidência científica. Revista Brasileira de Fisioterapia, v.11, n.1, p. 83-89, 2007.

SAMULSKI, D. In: Psicologia do esporte. Barueri: Manole, 2002.

Treinamento mental no tênis. Barueri: Manole, 2011

SANTOS, L.D. dos; AMARAL, L. Estudos Delphi com Q-Sort sobre a web: a sua utilização em sistemas de informação. In: CONFERÊNCIA DA ASSOCIAÇÃO PORTUGUESA DE SISTEMAS DE INFORMAÇÃO, 5., Lisboa, 2004. Actas.... Disponível em https://repositorium.sdum.uminho.pt/bitstream/1822/2280/1/ArtigoCAPSI2004Delphi.pdf. Acesso em: 8 nov. 2017. 
SHAPIRO, S. L. et al. Meditation and positive psychology. In.: SNYDER, C. R.; LOPEZ, S. J. (Eds.). Handbook of positive psychology, v. 5, n.1, p. 632-645, 2005.

SEKHON, B. S. Effects of progressive muscle relaxation and transcendental meditation treatment on rifle shooting performance. International Journal of Science and Research, v. 5, n. 4, p. 2035-2037, 2013.

Effects of transcendental meditation and progressive muscle relaxation treatment on pistol shooting performance. International Journal of Health, Sports and Physical Education, v. 2, n. 1, p. 39-41, 2013.

SILVA, L. N . O. et al. Cognição e esporte. Revista da Biologia, v.11, n. 1, p. 43-49, 2013.

SILVA, M. V. Estado mental flow e motivação no atletismo: dos velocistas aos ultramaratonistas. 2013. 67 f. Dissertação (Mestrado em Aspectos Biodinâmicos do Movimento Humano)- Faculdade Educação Física e Desporto, Universidade Federal de Juiz de Fora, Juiz de Fora, 2013.

SIN D.D., et al. Contemporary management of chronic obstructive pulmonary disease: scientific review. JAMA, v. 290, n.17, p.2301-12, 2003.

SLAGTER, H. A et al. Mental training affects distribution of limited brain resources. PLoS Biology, v.5, 2007.

SOLBERG, E. E. et al. The effects of long meditation on plasma melatonin and blood serotonin. Medicine Science Monitoring, v. 10, n.3, p. 96-101, 2004.

TRAVIS, F.; WALLACE, R. K. Autonomic and EEG patterns during eyes-closed rest and transcendental meditation (TM) practice: the basis for a neural model of TM practice. Consciousness and Cognition, v.8, p.302-318, 1999.

TUBINO, M. J. G. Estudos brasileiros sobre o esporte: ênfase no esporte-educação. Maringá: EDUEM, 2010.

VANDENBERGH, L.; ASSUNÇÃO, A. B. Concepções de mindfulness em Langer e KabatZinn: um encontro da ciência ocidental com a espiritualidade oriental. Contextos Clínicos, v.2, n. 2, p. 124-135, 2009.

VANDENBERGH, L.; SOUSA, A. C. Mindfulness nas terapias cognitivas e comportamentais. Revista Brasileira de Terapias Cognitivas, v. 2, n.1, p.35-44, 2006.

VERKHOSHANSHI, Y. V. Treinamento desportivo: teoria e metodologia. Porto Alegre: Artmed, 2001. (Coleção Kinesis).

WALLACE, R. K. et al. A wakeful hypometabolic physiologic state. American Journal of Physiology, v. 221, n.2, p.795-799, 1971.

WALlACE, R. K.; BEnSON, H. The physiology of meditation. Scientific American, v. 226, n. 2, p. 84-90, 1972. 
WEINBERG, R.; S. GOULD, D. In: Fundamentos da psicologia do esporte e do exercício. Porto Alegre: Artmed, 2001.

ZENG, H. Z. et al. The difference between anxiety and self-confidence between team and individual sports college varsity athletes. International Sports Journal, v. 13, n 4, p. 28-34, 2003.

Recebido em: 13/01/2016

Revisado em: 09/10/2017

Aprovado em: 26/11/2017

Endereço para correspondência:

paraovinicius@gmail.com

Vinícius Demarchi Silva Terra

Universidade Federal de São Paulo, Campus Baixada Santista.

Av. Alm. Saldanha da Gama, 89

Ponta da Praia

11030-400 - Santos, SP - Brasil 